\title{
3 Research Soure \\ Identification of minimal hepatic encephalopathy based on dynamic functional connectivity
}

\section{Yue Cheng}

Tianjin First Central Hospital

Gaoyan Zhang ( $\nabla$ zhanggaoyan_tg@163.com )

Tianjin University https://orcid.org/0000-0002-6791-9658

\section{Xiaodong Zhang}

Tianjin First Central Hospital

\section{Yuexuan Li}

Tianjin University

\section{Jingli Li}

tianjin first central hospital

Jiamin Zhou

Tianjin First Central Hospital

Lixiang Huang

tianjin first central hospital

\section{Shuangshuang Xie}

Tianjin First Central Hospital

\section{Wen Shen}

Tianjin First Central Hospital

\section{Research Article}

Keywords: dynamic functional connectivity, resting-state fMRI, minimal hepatic encephalopathy, machine learning, brain network

Posted Date: February 25th, 2021

DOl: https://doi.org/10.21203/rs.3.rs-196350/v1

License: (1) This work is licensed under a Creative Commons Attribution 4.0 International License. Read Full License

Version of Record: A version of this preprint was published at Brain Imaging and Behavior on March 23rd, 2021. See the published version at https://doi.org/10.1007/s11682-021-00468-x. 


\section{Abstract}

To investigate whether dynamic functional connectivity (DFC) metrics can better identify minimal hepatic encephalopathy (MHE) patients from cirrhotic patients without any hepatic encephalopathy (noHE) and healthy controls (HCs). Resting-state functional MRI data were acquired from 62 patients with cirrhosis (MHE, n=30; noHE, n=32) and $41 \mathrm{HCs}$. We used the sliding time window approach and functional connectivity analysis to extract the time-varying properties of brain connectivity. Three DFC characteristics (i.e., strength, stability, and variability) were calculated. For comparison, we also calculated the static functional connectivity (SFC). A linear support vector machine was used to differentiate MHE patients from noHE and HCs using DFC and SFC metrics as classification features. The leave-one-out cross-validation method was used to estimate the classification performance. The strength of DFC (DFC-Dstrength) achieved the best accuracy (MHE vs. noHE, 72.5\%; MHE vs. HCs, 84\%; and noHE vs. HCs, $88 \%$ ) compared to the other dynamic features. Compared to static features, the classification accuracies of the DFC-Dstrength feature were improved by $10.5 \%, 8 \%$, and $14 \%$ for MHE vs. noHE, MHE vs. $\mathrm{HC}$, and noHE vs. HCs, respectively. Based on the DFC-Dstrength, seven nodes were identified as the most discriminant features to classify MHE from noHE, including left inferior parietal lobule, left supramarginal gyrus, left calcarine, left superior frontal gyrus, left cerebellum, right postcentral gyrus, and right insula. In summary, DFC characteristics have a higher classification accuracy in identifying MHE from cirrhosis patients. Our findings suggest the usefulness of DFC in capturing neural processes and identifying disease-related biomarkers important for MHE identification.

\section{Introduction}

On the neurocognitive impairment severity continuum, minimal hepatic encephalopathy (MHE) is the mildest form and is considered to be the precursor to overt hepatic encephalopathy (OHE) (Hadjihambi, Arias, Sheikh, \& Jalan, 2018). MHE is defined as a condition in which cirrhotic patients have neuropsychiatric and neurophysiological defects despite normal mental status (San Martín-Valenzuela et al., 2020). In recent years, MHE has received considerable attention because of its association with a poor quality of life, decline in daily functioning, increased risk of traffic accidents, and a higher risk of progression to OHE (Labenz et al., 2019; Ridola, Nardelli, Gioia, \& Riggio, 2018; A. J. Wang et al., 2017). However, the diagnostic criteria for MHE have only recently been standardized, and it cannot be detected by routine clinical examinations. Therefore, there is an urgent need to identify reliable diagnostic biomarkers of MHE for preventative treatments and improvement of prognosis.

Numerous studies have explored MHE-related brain structural and functional abnormalities (Chen et al., 2020; Sato, 2019; Zhan, Chen, Gao, \& Zou, 2019; X. D. Zhang, Zhang, Wu, \& Lu, 2014). Chen et al. and Sun et al. demonstrated altered baseline brain activity measured by amplitude of low-frequency fluctuations and regional homogeneity in MHE (H. J. Chen et al., 2016; H. J. Chen, Zhu, Jiao, et al., 2012; H. J. Chen, Zhu, Yang, et al., 2012; Sun, Fan, Ye, \& Han, 2018). Remarkably, brain dysfunction is considered a core node in the progression of MHE. Qi et al. and Ye et al. found that cortical and interhemisphere dysconnectivity correlated with cognitive impairment in MHE (Qi, Zhang, Wu, et al., 2012; Ye et al., 2020). 
Furthermore, recent studies have focused on abnormal subnetwork integration in the dorsal attention network, default mode network (DMN), visual network, and auditory network (Qi, Xu, et al., 2012; Qi, Zhang, Xu, et al., 2012; Zhan et al., 2019; D. Zhang, Tu, Zhang, Jie, \& Lu, 2018; L. J. Zhang et al., 2012). These findings suggest the potential of resting state functional MRI (rs-fMRI) to provide biomarkers for the identification of MHE. However, all these previous studies implicitly assumed that the functional connectivity (FC) among brain regions is static and unchanging over the entire scanning period. The brain's neural landscape is a dynamic system, and there is preliminary evidence showing that timerelated patterns of FC (i.e., dynamic FC [DFC]) may provide more detailed information about brain disorders (van der Horn et al., 2020; Xue et al., 2020; Zhu et al., 2019). The DFC method can make up for the shortcomings of the "static" FC analysis, and it is possible to find more subtle changes in the early stages of the disease (Calhoun, Miller, Pearlson, \& Adali, 2014; Hutchison et al., 2013; Kucyi \& Davis, 2015). During the resting state and during the execution of restricted cognitive tasks, the brain network does exhibit FC changes within a few seconds, and these dynamic and time-varying changes in FC may help us in characterizing the behavioral and functional changes related to cognition. There have been some studies based on the dynamic hypotheses, i.e. to study the dynamic changes in the brain in patients with diseases, including autism, epilepsy, and schizophrenia (F. Liu et al., 2017; Mash et al., 2019; Sanfratello, Houck, \& Calhoun, 2019), which show that dynamic methods can capture more information over a period of time and find cognition. The related abnormal brain areas can more effectively describe abnormal changes in the disease.

Machine learning-based approaches can extract disease-related features and provide better predictive biomarkers for brain disorders. For example, a support vector machine (SVM), a typical machine learning method, has been used to distinguish cirrhotic patients from healthy controls (HCs) and predict disease severity with high accuracy (Feng Liu et al., 2015; Talpalaru, Bhagwat, Devenyi, Lepage, \& Chakravarty, 2019; G. Zhang, Cheng, \& Liu, 2017; Zhao, Ding, Du, Wang, \& Men, 2019). The aim of the current study was to explore whether MHE patients have abnormal dynamic FC characteristics and evaluate the identification accuracy of DFC as an MHE diagnostic biomarker. Therefore, we proposed a dynamic FC analysis to extract abnormal DFC characteristics and identify MHE from cirrhosis and HCs. Specifically, we also compare the identification capability of DFC with that of traditional static FC (SFC). We hypothesized that the dynamic characteristics of FC could improve the identification accuracy of MHE and offer a more effective diagnostic biomarker.

\section{Methods}

\subsection{Participants}

This study was approved by the Medical Research Ethics Committee of Tianjin First Central Hospital. A total of 62 patients with cirrhosis were recruited from our inpatient departments between September 2013 and March 2018. Written informed consent was obtained from all participants. The inclusion criteria were as follows: (1) patients with a diagnosis of cirrhosis, (2) being older than 18 years, (3) right-handedness, and (4) without any contraindication to MRI scanning. The diagnosis of liver cirrhosis was based on 
clinical history, biochemical findings, and imaging findings. Exclusion criteria for patient groups included history of any drug or alcohol abuse, history of OHE episodes, brain lesions such as tumor or stroke assessed on the basis of medical history and conventional MRI, and poor image quality with head motion of more than $2.0 \mathrm{~mm} /$ degree during MRI.

According to previous studies, the diagnosis of MHE was made according to two typical neuropsychological tests, number connection type A (NCT-A) and digit symbol test (DST) (Li et al., 2013; Weissenborn, Ennen, Schomerus, Ruckert, \& Hecker, 2001). Considering the effect of age and education level on the two neuropsychological scores, linear regression models were first constructed with regressors of age and education level and dependent variates of NCT-A and DST. The regressor coefficients were estimated in the HC group and were then used to predict the NCT-A and DST scores in patient groups. When at least one of the differences between predicted and true values was above two standard deviations of the mean value in $\mathrm{HC}$ groups, the cirrhosis patients were regarded as having MHE. According to this criterion, 30 of the 62 patients were diagnosed with $\mathrm{MHE}$, and the rest were assigned to the noHE group.

Forty-one age-and sex-matched HCs were recruited from the local community. All HCs had no history of liver disease or other systemic diseases, and they underwent neuropsychological tests before the MR scan.

\subsection{Laboratory tests}

Laboratory parameters, including blood ammonia, albumin, total bilirubin, and prothrombin time, were obtained for all patients during the week before MRI to assess the severity of cirrhosis. The Child-Pugh score was used to assess liver function. No blood samples were collected from any HCs.

\subsection{MRI Data Acquisition}

All imaging data were acquired using a 3.0 Tesla MRI scanner (TIM-Trio, Siemens Medical Solutions, Erlangen, Germany). Foam padding was used to reduce head motion. For rs-fMRI, 200 T2*-weighted volumes were acquired with slices aligned to the anterior commissure (AC)-posterior commissure (PC) plane (TR, $2500 \mathrm{~ms}$; TE, $30 \mathrm{~ms}$; FOV, $220 \mathrm{~mm} \times 220 \mathrm{~mm}$; matrix, 96×96; slice thickness, $3 \mathrm{~mm}$; and slice gap, $0.3 \mathrm{~mm}$ for 40 slices). The subjects were instructed to keep their eyes closed and stay awake during scanning.

\subsection{Functional MRI Image preprocessing}

Preprocessing was performed using the Gretna toolbox (https://www.nitrc.org/projects/gretna/) implemented in MATLAB (version 2013a; MathWorks, Natick, MA, USA). The first 10 volumes were excluded to ensure steady-state longitudinal magnetization, and the remaining 190 images were corrected for slice acquisition times and head movements. Subjects with more than $2 \mathrm{~mm}$ maximum displacement or with more than $2^{\circ}$ of angular rotation were excluded. The functional images were then 
spatially normalized to the Montreal Neurological Institute space using an echo planar imaging template ( $3 \times 3 \times 3 \mathrm{~mm}$ isotropic voxels) (Calhoun et al., 2017) and smoothed using a $6 \mathrm{~mm}$ full width at half maximum (FWHM) kernel. After removal of linear trends, fMRI data were band-pass filtered (0.01-0.08 $\mathrm{Hz}$ ) to reduce the very-low-frequency drift and high-frequency physiological noise. Finally, nuisance covariates, including six motion parameters, cerebrospinal fluid, and white matter signals, were regressed out to further reduce the effects of confounding factors.

\subsection{SFC analyses}

FC analyses were performed on the SFC of patients and HCs using the Gretna toolbox (https://www.nitrc.org/projects/gretna/). First, we employed a 264-node functional atlas (Power et al., 2011) for node definition. Then, the time course of each node was extracted by averaging the time courses of all voxels therein. The strength of the functional connection is expressed as the Pearson correlation coefficient between the time courses of any two nodes. Then, the correlation matrix is Ztransformed using Fisher' $s$ transformation. SFCs larger than 0.35 were selected to construct the brain network. The node degree is obtained by summing the number of connections that link this node to the rest of the network.

$$
\operatorname{SFC}(i)=D_{z(i)}
$$

\section{where $D_{z(i)}$ is the total z-transformed SFC connections to node i (i.e. degree of node}

i).

\subsection{DFC analyses}

The DFC was estimated using a sliding temporal window with a width of 50 TRs (125 s) and steps of 1 TR $(2.5 \mathrm{~s})$. The node definition was consistent with the SFC analysis section. For each sliding window, a pairwise Pearson correlation was used to obtain the FC matrix in each window with a threshold of 0.35 . Therefore, DFC was defined as the FC matrix series.

To quantitatively describe the time-varying characteristics of the DFC, three measurements were calculated for each node, including the overall strength of the nodal degree fluctuation with time (DFCDstrength), stability of nodal degree fluctuation between adjacent windows (DFC-Dstability), and overall variation of the nodal degree fluctuation over time (DFC-Dvariance) (J. Liu, Liao, Xia, \& He, 2018). 


$$
\begin{aligned}
& \text { DFC-Dstrength (i) }=\frac{1}{T} \sum_{t=1}^{T} D_{z(i) t} \\
& \text { DFC-Dstability (i) }=1-\frac{1}{T-1} \sum_{t=1}^{T-1} \frac{\left|D_{z(i)(t+1)}-D_{z(i) t}\right|}{2} \\
& \text { DFC-Dvariance (i) }=\frac{1}{T} \sum_{t=1}^{T}\left(D_{z(i) t}-\overline{D_{z(l)}}\right)^{2}
\end{aligned}
$$

where $D_{z(i) t}$ is the node degree of the $\mathrm{z}$ - transformed DFC of node $\mathrm{i}$ in window $\mathrm{t}$,

\section{and $\mathrm{T}$ is the total number of time windows. For visualization, see Fig 1.}

\subsection{Classification analysis based on DFC and SFC}

This study used the Library for Support Vector Machines (LIBSVM) toolkit (http://www.csie.ntu.edu.tw/ cjlin/libsvm/) to conduct the SVM classification process for each SFC and DFC measurement. Same procedure and classifier parameters were used for SFC and DFC based discrimination. Considering that some features are noninformative or redundant, the F-score was adopted as the feature selection method, and an SVM with a radius basis function as the kernel and a cost coefficient of 15 was used as the classifier. A leave-one-out cross-validation strategy was applied to construct the classifier. In each trial, the samples were divided into a training set ( $\mathrm{k}-1$ samples) and a test set (the remaining one sample) (F. Liu, Wee, Chen, \& Shen, 2014). Based on the k-1 samples, the input feature was selected using an F-score method, which calculated the between-group differences, significant at $p<0.05$. Then, SVM was used to classify (a) MHE versus HCs, (b) noHE versus HCs, and (c) MHE versus noHE to construct discrimination maps. Consistent features selected in all folds were defined as discriminative features. As the classifier may be ill-trained, we performed nonparametric permutation tests $(1,000$ relabeling) to estimate the statistical significance of the classification accuracy at a significance level of $p<0.05$.

\subsection{Correlation analysis}

After comparing the classification ability of different measurements in discriminating MHE from noHE, we could obtain the nodes with the most discriminative features based on the strongest classification ability. Then, the relationship between the neuropsychological test and nodal measurement was assessed in all cirrhotic patients using a linear regression model with sex, age, education level, and head movement (FD) as covariant. $\mathrm{P}<0.05$ (two-tailed) was considered to be statistically significant.

\section{Results}

Table 1 shows the demographic and clinical characteristics of the participants. There were no significant differences in terms of age, sex, or education among the MHE, noHE, and HCs. The neuropsychological performance of MHE patients was significantly worse than that of noHE patients and HCs. 
As shown in Fig. 2, the DFC-Dstrength achieved the best accuracy (MHE vs. noHE, 72.5\%; MHE vs. HCs, 84\%; and noHE vs. HCs, 88\%) than the other dynamic features. Compared to static features, the classification accuracies of the DFC-Dstrength feature were improved by $10.5 \%, 8 \%$, and $14 \%$ for MHE vs. noHE, MHE vs. HC, and noHE vs. HCs, respectively. For the best accuracy with DFC-Dstrength, a permutation test with 1,000 trials of random labels was conducted, and the results showed that all three classifications were significant $(p<0.05)$.

Moreover, based on the DFC-Dstrength, seven nodes were identified as the most discriminant features to classify MHE from noHE, including left inferior parietal lobule (IPL), left supramarginal gyrus (SMG), left calcarine, left superior frontal gyrus (SFG), left cerebellum, right postcentral gyrus (PCG), and right insula (see Fig 3).

After that, a regression model was constructed using the DFC-Dstrength in the seven nodes as regressors, NCT-A as the dependent variable, and sex, age, education level, and head motion as covariates to calculate the correlation between discriminative DFC-Dstrength and neuropsychological level. It is found that the DFC-Dstrength values in the right insula was positively correlated with NCT-A score in the patient group ( $p=0.002$; see Table 2). No significant correlation was found between the DFC-Dstrength in other nodes and NCT-A score or between other SFC/DFC measurements and DST scores.

\section{Discussion}

In this study, we applied a machine learning method to identify MHE from noHE and HCs based on wholebrain DFC. We compared the dynamic and static methods and found that the classification accuracies of DFC features were significantly higher than that of SFC, which indicates the usefulness of DFC in capturing the neural process and finding disease-related biomarkers important for MHE identification. Several regions were identified as discriminative features; these regions were located mainly in the frontal and parietal lobes, and were correlated with neuropsychological scores in cirrhotic patients.

Our study proposes a new neuroimaging biomarker for the detection of MHE. As we know, MHE has the potential to progress into OHE, which can cause irreversible brain damage (Tapper et al., 2020). Studies have shown that MHE can be reversed by prompt treatment (Abid et al., 2020; Butterworth \& McPhail, 2019; Nardone et al., 2016); therefore, it is important to diagnose and treat MHE before major neurological damage occurs. Currently, various neuropsychological tests are used as the diagnostic standard for $\mathrm{MHE}$, but they are easily affected by age, education level, and mental state. Neuroimaging biomarkers have the advantage of reliability and objectivity, which can be a powerful supplement to neuropsychological tests. SVM is an efficient classifier that can extract disease-related features and can be used as a diagnostic tool. Some researchers have tried to find neuroimaging biomarkers of MHE using the SVM method. For example, Chen et al. identified MHE from noHE with a relatively high accuracy by measuring spontaneous brain activity (H. J. Chen et al., 2016; Q. F. Chen, Chen, Liu, Sun, \& Shen, 2016). However, these studies were based on static connectivity measures. Accumulating evidence indicates that DFC can capture abnormal alterations more accurately in brain disorders, such as studies on mild cognitive impairment 
and Parkinson's disease (Cai et al., 2019; X. Chen et al., 2017). To verify this statement, we compared the diagnostic accuracy of DFC with SFC. Our results showed that DFC-Dstrength performed best among the three DFC metrics, indicating that the strength, but not the variance, of functional connection can better discriminate individual patients. Although static FC also reflects the FC strength, it is calculated for the entire time duration. Comparing the SFC-Dstrength with DFC-Dstrength, the latter can obviously improve classification accuracies when distinguishing MHE vs. noHE, MHE vs. HCs, and noHE vs. HCs by $10.5 \%$, $8 \%$, and $14 \%$, respectively. This result shows that DFC analysis can provide a better representation of cirrhotic disease by determining the time-varying strength characteristics. Moreover, the relationship between DFC metrics (DFC-Dstrength) and neuropsychological scores (the clinical marker of MHE) in our study shows that the DFC-Dstrength can reflect the cognitive changes, providing a new perspective to understand cirrhotic disease.

Using the criterion of the best diagnostic accuracy (DFC-Dstrength), we identified seven regions associated with MHE dysfunction. These regions included the left IPL, SMG, calcarine, SFG, cerebellum and right PCG, and insula. Most of these regions have been reported in previous studies on MHE. The findings for each region are described below.

Attention deficit and executive function decline are early manifestations of MHE. Given that the DMN is a major contributor to normal cognitive functions, such as attention and executive functions, decreased connectivity in the IPL and SMG (major posterior components of the DMN) is identified in MHE. Significant FC reduction within the DMN is associated with cognitive impairment due to MHE. Consistent with our findings, previous $\mathrm{fMRI}$ studies revealed significant FC reduction within the DMN in patients with MHE, which was associated with cognitive impairment as well as hepatic encephalopathy development. Qi et al. reported that FC disturbance within the DMN occurred earlier than that of structural connections in MHE patients, and they believed that FC was a sensitive index for detecting early abnormalities of MHE (Qi, Xu, et al., 2012).

Deficits in visual information processing, such as deficits in visual memory, visuomotor, and visuospatial integration, are also regarded as characteristics of MHE (Bajaj, Wade, \& Sanyal, 2009). Task-dependent functional MRI has revealed the MHE-related neural mechanism underlying impaired visual judgment (Zafiris et al., 2004). Many rs-fMRI studies have described decreased neuronal activity or connectivity in the visual cortex in cirrhotic patients with MHE (Cheng et al., 2018; M. Wang et al., 2019). Calcarine is a key node of the ventral visual pathway (Borra \& Rockland, 2011), and abnormalities in calcarine may be related to impaired visual-spatial capabilities. Therefore, it is appropriate to determine calcarine as a discriminative region for MHE in our study.

In addition, some regions with increased FC were also selected as classification features for MHE in the present study, including the left cerebellum, right PCG, and right insula. The enhanced functional connection may reflect a compensatory mechanism for functional damage in the high-level cognition and visual regions in MHE. A similar compensatory phenomenon has also been reported in previous studies. Hitherto, we reported that FC was reduced in hub regions and increased in non-hub regions, including the 
PCG and cerebellum (Cheng et al., 2018; G. Zhang et al., 2017). Consistent with our findings, Chen et al. and Qi et al. also found increased brain activity in the cerebellum (H. J. Chen, Zhu, Jiao, et al., 2012; Qi, Zhang, Wu, et al., 2012). During the flicker light test, $\mathrm{fMRI}$ revealed that MHE patients showed enhanced coupling between the IPL and PCG (Zafiris et al., 2004). A compensatory role of the insula was highlighted by QI et al., who believed that the insula played an important role in the progression of hepatic encephalopathy (Qi, Zhang, Wu, et al., 2012). Herein, we demonstrated the increased FC of the insula in $\mathrm{MHE}$, the mildest form of $\mathrm{HE}$, which may implicate the advantage of dynamic metrics in detecting early brain alterations. The positive correlation between DFC values in this region and NCT-A, a test for psychomotor function, also provided supporting evidence for this implication.

There are several limitations to our study. First, the sample size was relatively small, which may have reduced the generalizability of our results. Second, the heterogeneous etiology of the recruited patients in our study may have produced bias in the classification results, because the brain damage caused by liver cirrhosis with different etiologies can also be slightly different. Third, we only used time-varying connectivity metrics (nodal degree) to identify MHE patients; DFC changes of brain intrinsic networks and multimodal analysis of neuroimaging datasets may further improve classification performance.

\section{Conclusions}

In conclusion, by integrating dynamic functional brain connections and SVM, this study provides new biomarkers for the accurate diagnosis of MHE. The generated biomarkers can assist clinicians in the early diagnosis and treatment of MHE. Furthermore, the identified brain regions may help localize the etiological origin and understand the pathogenesis of MHE.

\section{Declarations}

\section{Acknowledgements}

\section{Author contributions}

YC designed the study and wrote the manuscript; G.-Y. Z proposed the data analysis frame, analyzed the data and revised the manuscript; $X Z$ collected the data and did some data analysis, $Y L$ and JL did some data processing and visualization; JZ and LH collected the data and did some statistical analysis; SX and WS drafted and revised the manuscript. We would like to thank Editage (www.editage.cn) for English language editing.

\section{Funding Sources}

The study was supported by grants from National Natural Science Foundation of China (No. 81601482, No. 61876126, No. 81901710 and No. 81701679).

\section{Consent to participate}


Informed consent was obtained from all participants included in the study

\section{Consent for publication}

Not applicable

\section{Availability of data and material}

Not applicable

\section{Code availability}

Not applicable

\section{Compliance with ethical standards}

\section{Conflict of Interest}

The authors have no conflicts of interest to declare.

\section{Ethical approval}

All procedures were in accordance with the ethical standards of the institutional and/or national research committee and with the 1964 Helsinki declaration and its later amendments or comparable ethical standards.

\section{References}

Abid, S., Kamran, M., Abid, A., Butt, N., Awan, S., \& Abbas, Z. (2020). Minimal Hepatic Encephalopathy: Effect of H. pylori infection and small intestinal bacterial overgrowth treatment on clinical outcomes. Sci Rep, 10(1), 10079. doi:10.1038/s41598-020-67171-7

Bajaj, J. S., Wade, J. B., \& Sanyal, A. J. (2009). Spectrum of neurocognitive impairment in cirrhosis: Implications for the assessment of hepatic encephalopathy. Hepatology, 50(6), 2014-2021. doi:10.1002/hep.23216

Borra, E., \& Rockland, K. S. (2011). Projections to early visual areas v1 and v2 in the calcarine fissure from parietal association areas in the macaque. Front Neuroanat, 5, 35. doi:10.3389/fnana.2011.00035

Butterworth, R. F., \& McPhail, M. J. W. (2019). L-Ornithine L-Aspartate (LOLA) for Hepatic Encephalopathy in Cirrhosis: Results of Randomized Controlled Trials and Meta-Analyses. Drugs, 79(Suppl 1), 31-37. doi:10.1007/s40265-018-1024-1

Cai, J., Liu, A., Mi, T., Garg, S., Trappe, W., McKeown, M. J., \& Wang, Z. J. (2019). Dynamic Graph Theoretical Analysis of Functional Connectivity in Parkinson's Disease: The Importance of Fiedler Value. 
Calhoun, V. D., Miller, R., Pearlson, G., \& Adali, T. (2014). The Chronnectome: Time-Varying Connectivity Networks as the Next Frontier in fMRI Data Discovery. Neuron, 84(2), 262-274.

doi:10.1016/j.neuron.2014.10.015

Calhoun, V. D., Wager, T. D., Krishnan, A., Rosch, K. S., Seymour, K. E., Nebel, M. B., . . Kiehl, K. (2017). The impact of T1 versus EPI spatial normalization templates for fMRI data analyses. Hum Brain Mapp, 38(11), 5331-5342. doi:10.1002/hbm.23737

Chen, H. J., Zhang, L., Jiang, L. F., Chen, Q. F., Li, J., \& Shi, H. B. (2016). Identifying minimal hepatic encephalopathy in cirrhotic patients by measuring spontaneous brain activity. Metab Brain Dis, 31(4), 761-769. doi:10.1007/s11011-016-9799-9

Chen, H. J., Zhu, X. Q., Jiao, Y., Li, P. C., Wang, Y., \& Teng, G. J. (2012). Abnormal baseline brain activity in low-grade hepatic encephalopathy: a resting-state fMRI study. J Neurol Sci, 318(1-2), 140-145. doi:10.1016/j.jns.2012.02.019

Chen, H. J., Zhu, X. Q., Yang, M., Liu, B., Zhang, Y., Wang, Y., \& Teng, G. J. (2012). Changes in the regional homogeneity of resting-state brain activity in minimal hepatic encephalopathy. Neurosci Lett, 507(1), 5-9. doi:10.1016/j.neulet.2011.11.033

Chen, L. H., Shi, J. Y., Zou, T. X., Zhang, L., Gou, Y. P., Lin, Y. Q., \& Chen, H. J. (2020). Disturbance of thalamic metabolism and its association with regional neural dysfunction and cognitive impairment in minimal hepatic encephalopathy. European Journal of Radiology, 131. doi:10.1016/j.ejrad.2020.109252

Chen, Q. F., Chen, H. J., Liu, J., Sun, T., \& Shen, Q. T. (2016). Machine Learning Classification of Cirrhotic Patients with and without Minimal Hepatic Encephalopathy Based on Regional Homogeneity of Intrinsic Brain Activity. PLoS One, 11(3), e0151263. doi:10.1371/journal.pone.0151263

Chen, X., Zhang, H., Zhang, L., Shen, C., Lee, S. W., \& Shen, D. (2017). Extraction of dynamic functional connectivity from brain grey matter and white matter for $\mathrm{MCl}$ classification. Hum Brain Mapp, 38(10), 5019-5034. doi:10.1002/hbm.23711

Cheng, Y., Zhang, G., Shen, W., Huang, L. X., Zhang, L., Xie, S. S., . . Liu, B. (2018). Impact of previous episodes of hepatic encephalopathy on short-term brain function recovery after liver transplantation: a functional connectivity strength study. Metab Brain Dis, 33(1), 237-249. doi:10.1007/s11011-017-0155-5

Hadjihambi, A., Arias, N., Sheikh, M., \& Jalan, R. (2018). Hepatic encephalopathy: a critical current review. Hepatol Int, 12(Suppl 1), 135-147. doi:10.1007/s12072-017-9812-3

Hutchison, R. M., Womelsdorf, T., Allen, E. A., Bandettini, P. A., Calhoun, V. D., Corbetta, M., . . Chang, C. (2013). Dynamic functional connectivity: Promise, issues, and interpretations. Neuroimage, 80, 360-378. doi:10.1016/j.neuroimage.2013.05.079 
Kucyi, A., \& Davis, K. D. (2015). The dynamic pain connectome. Trends in Neurosciences, 38(2), 86-95. doi:10.1016/j.tins.2014.11.006

Labenz, C., Toenges, G., Schattenberg, J. M., Nagel, M., Sprinzl, M. F., Nguyen-Tat, M., . . Worns, M. A. (2019). Clinical Predictors for Poor Quality of Life in Patients With Covert Hepatic Encephalopathy. J Clin Gastroenterol, 53(7), e303-e307. doi:10.1097/MCG.0000000000001149

Li, S. W., Wang, K., Yu, Y. Q., Wang, H. B., Li, Y. H., \& Xu, J. M. (2013). Psychometric hepatic encephalopathy score for diagnosis of minimal hepatic encephalopathy in China. World Journal of Gastroenterology, 19(46), 8745-8751. doi:10.3748/wjg.v19.i46.8745

Liu, F., Guo, W., Fouche, J.-P., Wang, Y., Wang, W., Ding, J., . . Chen, H. (2015). Multivariate classification of social anxiety disorder using whole brain functional connectivity. Brain Structure and Function, 220(1), 101-115. doi:10.1007/s00429-013-0641-4

Liu, F., Wang, Y. F., Li, M. L., Wang, W. Q., Li, R., Zhang, Z. Q., . . Chen, H. F. (2017). Dynamic functional network connectivity in idiopathic generalized epilepsy with generalized tonic-clonic seizure. Human Brain Mapping, 38(2), 957-973. doi:10.1002/hbm.23430

Liu, F., Wee, C. Y., Chen, H., \& Shen, D. (2014). Inter-modality relationship constrained multi-modality multitask feature selection for Alzheimer's Disease and mild cognitive impairment identification. Neuroimage, 84, 466-475. doi:10.1016/j.neuroimage.2013.09.015

Liu, J., Liao, X., Xia, M., \& He, Y. (2018). Chronnectome fingerprinting: Identifying individuals and predicting higher cognitive functions using dynamic brain connectivity patterns. Hum Brain Mapp, 39(2), 902-915. doi:10.1002/hbm.23890

Mash, L. E., Linke, A. C., Olson, L. A., Fishman, I., Liu, T. T., \& Muller, R. A. (2019). Transient states of network connectivity are atypical in autism: A dynamic functional connectivity study. Human Brain Mapping, 4O(8), 2377-2389. doi:10.1002/hbm.24529

Nardone, R., Taylor, A. C., Holler, Y., Brigo, F., Lochner, P., \& Trinka, E. (2016). Minimal hepatic encephalopathy: A review. Neurosci Res, 111, 1-12. doi:10.1016/j.neures.2016.04.009

Power, J. D., Cohen, A. L., Nelson, S. M., Wig, G. S., Barnes, K. A., Church, J. A., . . Petersen, S. E. (2011). Functional network organization of the human brain. Neuron, 72(4), 665-678. doi:10.1016/j.neuron.2011.09.006

Qi, R., Xu, Q., Zhang, L. J., Zhong, J., Zheng, G., Wu, S., . . Lu, G. (2012). Structural and functional abnormalities of default mode network in minimal hepatic encephalopathy: a study combining DTI and fMRI. PLoS One, 7(7), e41376. doi:10.1371/journal.pone.0041376

Qi, R., Zhang, L., Wu, S., Zhong, J., Zhang, Z., Zhong, Y., . . Lu, G. (2012). Altered resting-state brain activity at functional MR imaging during the progression of hepatic encephalopathy. Radiology, 264(1), 
Qi, R., Zhang, L. J., Xu, Q., Zhong, J., Wu, S., Zhang, Z., . . Lu, G. (2012). Selective impairments of restingstate networks in minimal hepatic encephalopathy. PLoS One, 7(5), e37400.

doi:10.1371/journal.pone.0037400

Ridola, L., Nardelli, S., Gioia, S., \& Riggio, O. (2018). Quality of life in patients with minimal hepatic encephalopathy. World J Gastroenterol, 24(48), 5446-5453. doi:10.3748/wjg.v24.i48.5446

San Martín-Valenzuela, C., Borras-Barrachina, A., Gallego, J. J., Urios, A., Mestre-Salvador, V., CorreaGhisays, P., ... Montoliu, C. (2020). Motor and Cognitive Performance in Patients with Liver Cirrhosis with Minimal Hepatic Encephalopathy. J Clin Med, 9(7). doi:10.3390/jcm9072154

Sanfratello, L., Houck, J. M., \& Calhoun, V. D. (2019). Dynamic Functional Network Connectivity in Schizophrenia with Magnetoencephalography and Functional Magnetic Resonance Imaging: Do Different Timescales Tell a Different Story? Brain Connectivity, 9(3), 251-262. doi:10.1089/brain.2018.0608

Sato, T. (2019). Decreased Mean Kurtosis in the Putamen Is a Diagnostic Feature of Minimal Hepatic Encephalopathy in Patients With Cirrhosis. American Journal of Gastroenterology, 114, S14-S15. doi:10.14309/01.ajg.0000582088.65696.b5

Sun, Q., Fan, W. L., Ye, J., \& Han, P. (2018). Abnormal Regional Homogeneity and Functional Connectivity of Baseline Brain Activity in Hepatitis B Virus-Related Cirrhosis With and Without Minimal Hepatic Encephalopathy. Frontiers in Human Neuroscience, 12. doi:10.3389/fnhum.2018.00245

Talpalaru, A., Bhagwat, N., Devenyi, G. A., Lepage, M., \& Chakravarty, M. M. (2019). Identifying schizophrenia subgroups using clustering and supervised learning. Schizophr Res, 214, 51-59. doi:10.1016/j.schres.2019.05.044

Tapper, E. B., Zhao, L., Nikirk, S., Baki, J., Parikh, N. D., Lok, A. S., \& Waljee, A. K. (2020). Incidence and Bedside Predictors of the First Episode of Overt Hepatic Encephalopathy in Patients With Cirrhosis. Am J Gastroenterol, 115(12), 2017-2025. doi:10.14309/ajg.0000000000000762

van der Horn, H. J., Vergara, V. M., Espinoza, F. A., Calhoun, V. D., Mayer, A. R., \& van der Naalt, J. (2020). Functional outcome is tied to dynamic brain states after mild to moderate traumatic brain injury. Hum Brain Mapp, 41(3), 617-631. doi:10.1002/hbm.24827

Wang, A. J., Peng, A. P., Li, B. M., Gan, N., Pei, L., Zheng, X. L., . . Zhu, X. (2017). Natural history of covert hepatic encephalopathy: An observational study of 366 cirrhotic patients. World J Gastroenterol, 23(34), 6321-6329. doi:10.3748/wjg.v23.i34.6321

Wang, M., Cui, J., Liu, Y., Zhou, Y., Wang, H., Wang, Y., . . Yu, Y. (2019). Structural and functional abnormalities of vision-related brain regions in cirrhotic patients: a MRI study. Neuroradiology, 61(6), 695702. doi:10.1007/s00234-019-02199-9 
Weissenborn, K., Ennen, J. C., Schomerus, H., Ruckert, N., \& Hecker, H. (2001). Neuropsychological characterization of hepatic encephalopathy. Journal of Hepatology, 34(5), 768-773. doi:Doi 10.1016/S0168-8278(01)00026-5

Xue, K., Liang, S., Yang, B., Zhu, D., Xie, Y., Qin, W., .. Y Yu, C. (2020). Local dynamic spontaneous brain activity changes in first-episode, treatment-naive patients with major depressive disorder and their associated gene expression profiles. Psychol Med, 1-10. doi:10.1017/S0033291720003876

Ye, M., Guo, Z., Li, Z. P., Lin, X. S., Li, J., Jiang, G. H., . . Lv, X. F. (2020). Aberrant inter-hemispheric coordination characterizes the progression of minimal hepatic encephalopathy in patients with HBVrelated cirrhosis. Neuroimage-Clinical, 25. doi:10.1016/j.nicl.2020.102175

Zafiris, O., Kircheis, G., Rood, H. A., Boers, F., Haussinger, D., \& Zilles, K. (2004). Neural mechanism underlying impaired visual judgement in the dysmetabolic brain: an fMRI study. Neuroimage, 22(2), 541552. doi:10.1016/j.neuroimage.2004.01.038

Zhan, C. Y., Chen, H. J., Gao, Y. Q., \& Zou, T. X. (2019). Functional Network-Based Statistics Reveal Abnormal Resting-State Functional Connectivity in Minimal Hepatic Encephalopathy. Frontiers in Neurology, 10. doi:10.3389/fneur.2019.00033

Zhang, D., Tu, L., Zhang, L. J., Jie, B., \& Lu, G. M. (2018). Subnetwork mining on functional connectivity network for classification of minimal hepatic encephalopathy. Brain Imaging Behav, 12(3), 901-911. doi:10.1007/s11682-017-9753-4

Zhang, G., Cheng, Y., \& Liu, B. (2017). Abnormalities of voxel-based whole-brain functional connectivity patterns predict the progression of hepatic encephalopathy. Brain Imaging Behav, 11(3), 784-796. doi:10.1007/s11682-016-9553-2

Zhang, L. J., Zheng, G., Zhang, L., Zhong, J., Wu, S., Qi, R., . . Lu, G. (2012). Altered brain functional connectivity in patients with cirrhosis and minimal hepatic encephalopathy: a functional MR imaging study. Radiology, 265(2), 528-536. doi:10.1148/radiol.12120185

Zhang, X. D., Zhang, L. J., Wu, S. Y., \& Lu, G. M. (2014). Multimodality magnetic resonance imaging in hepatic encephalopathy: an update. World J Gastroenterol, 20(32), 11262-11272.

doi:10.3748/wjg.v20.i32.11262

Zhao, J., Ding, X., Du, Y., Wang, X., \& Men, G. (2019). Functional connectivity between white matter and gray matter based on fMRI for Alzheimer's disease classification. Brain Behav, 9(10), e01407. doi:10.1002/brb3.1407

Zhu, Y., Qi, S., Zhang, B., He, D., Teng, Y., Hu, J., \& Wei, X. (2019). Connectome-Based Biomarkers Predict Subclinical Depression and Identify Abnormal Brain Connections With the Lateral Habenula and Thalamus. Front Psychiatry, 10, 371. doi:10.3389/fpsyt.2019.00371 


\section{Tables}

Table 1. Demographic, Neuropsychological and Clinical Data

\begin{tabular}{lcccc}
\hline & MHE(n=30) & noHE $(\mathrm{n}=32)$ & $\mathrm{HC}(\mathrm{n}=41)$ & $\mathrm{P}$ \\
\hline Sex(male/female) & $21 / 9$ & $18 / 14$ & $28 / 13$ & $0.449^{\dagger}$ \\
Age(years) & $50.9 \pm 6.3$ & $47.9 \pm 8.2$ & $50.1 \pm 7.3$ & $0.153^{\ddagger}$ \\
\hline Education level (years) & $12.3 \pm 3.0$ & $12.3 \pm 3.5$ & $13.0 \pm 2.6$ & $0.499^{\ddagger}$ \\
\hline Neuropsychological tests & & & & \\
NCT-A(seconds) & $78.9 \pm 14.6$ & $43.1 \pm 12.7$ & $41.6 \pm 3.2$ & $<0.001^{\S}$ \\
\hline & & & & $<0.001^{\|}$ \\
\hline DST(score) & & & & $0.599^{\Uparrow}$ \\
\hline & $30.1 \pm 11.7$ & $43.1 \pm 9.5$ & $47.9 \pm 10.2$ & $<0.001^{\S}$ \\
\hline Laboratory examination & & & & $<0.001^{\|}$ \\
\hline Prothrombin time(seconds) & $18.8 \pm 5.2$ & $16.8 \pm 6.0$ & - & $0.105^{\S}$ \\
\hline Albumin(mg/dl) & $30.0 \pm 5.9$ & $32.0 \pm 5.8$ & - & $0.565^{\S}$ \\
\hline Total bilirubin(mg/dl) & $106.4 \pm 170$ & $96.1 \pm 139.3$ & - & $0.737^{\S}$ \\
Blood ammonia(in mod/L) & $72.6 \pm 31.5$ & $55.1 \pm 21.3$ & - & $0.009^{\S}$ \\
\hline Child-Pugh score (A/B/C) & $1 / 7 / 22$ & $5 / 16 / 11$ & - & \\
\hline
\end{tabular}

Data are presented as mean \pm standard deviation.

†: Pearson $\chi^{2}$ test of three groups (two-tailed), ‡: One-way analysis of variance test among three groups (two-tailed), §: Two-sample t test between MHE and noHE groups (two-tailed), \|: Two-sample t test between MHE and HC groups (two-tailed), I: Two-sample t test between noHE and HC groups (twotailed).

Abbreviations: DST $=$ digit-symbol test, $\mathrm{HC}=$ healthy control, $\mathrm{MHE}=$ mild hepatic encephalopathy, NCT$\mathrm{A}=$ number connection test of type $\mathrm{A}, \mathrm{noHE}=$ cirrhotic patients without clinical hepatic encephalopathy .

Table 2. Significance of DFC-Dstrength in seven discriminative nodes with NCT-A score in the regression model

\begin{tabular}{lllll}
\hline Regressors & MNI coordinates & Beta coefficients & T-value & P-value \\
\hline R. postcentral gyrus & $(13,-33,75)$ & 0.026 & 0.236 & 0.812 \\
L. pinferior parietal lobule & $(-29,-43,61)$ & -0.128 & -0.778 & 0.440 \\
R. insular & $(36,-9,14)$ & 0.470 & 3.335 & $0.002 *$ \\
L. supramarginal gyrus & $(-60,-25,14)$ & -0.164 & -1.243 & 0.219 \\
L. calcarine & $(-8,-81,7)$ & -0.237 & -1.691 & 0.097 \\
L. superior frontal gyrus & $(-21,41,-20)$ & 0.140 & 0.815 & 0.419 \\
L. cerebellum & $(1,-62,-18)$ & 0.127 & 1.083 & 0.284 \\
\hline
\end{tabular}

\section{Abbreviations: MNI, Montreal Neurological Institute}

\section{Figures}




\section{Whole brain DFC matrices}

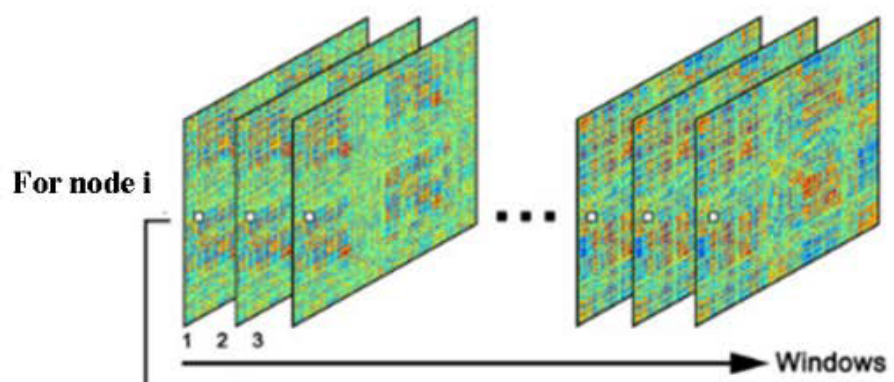

node degree time series
Dorsal attention

Cerebellar

Subcortical

Salience

Fronto-parietal Task

Visual

Ventral_atten

Memory retrieval

Default mode

Auditory

Cingulo-opercular Task

Sensory/Somatomotor Mouth

Sensory/Somatomotor Hand

Uncertain

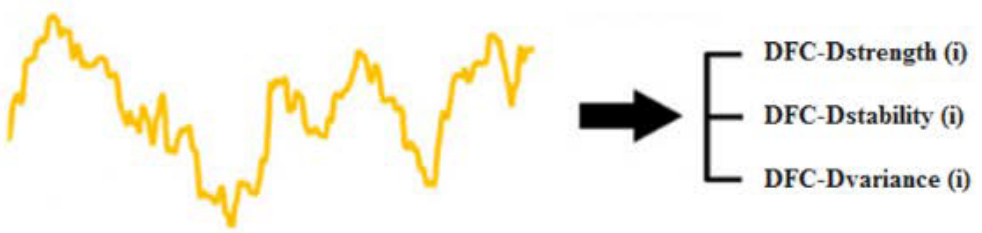

\section{Figure 1}

The visualization of DFC measurements. Left: Transient dynamic functional connectivity matrices at different sliding windows. The strength of functional connection is expressed as the Pearson correlation coefficient between the time courses of any two nodes, which fluctuates across windows. Right: definition of nodes. The regions of interest (ROI) set including 264 regions was obtained from a previously defined functional atlas. 


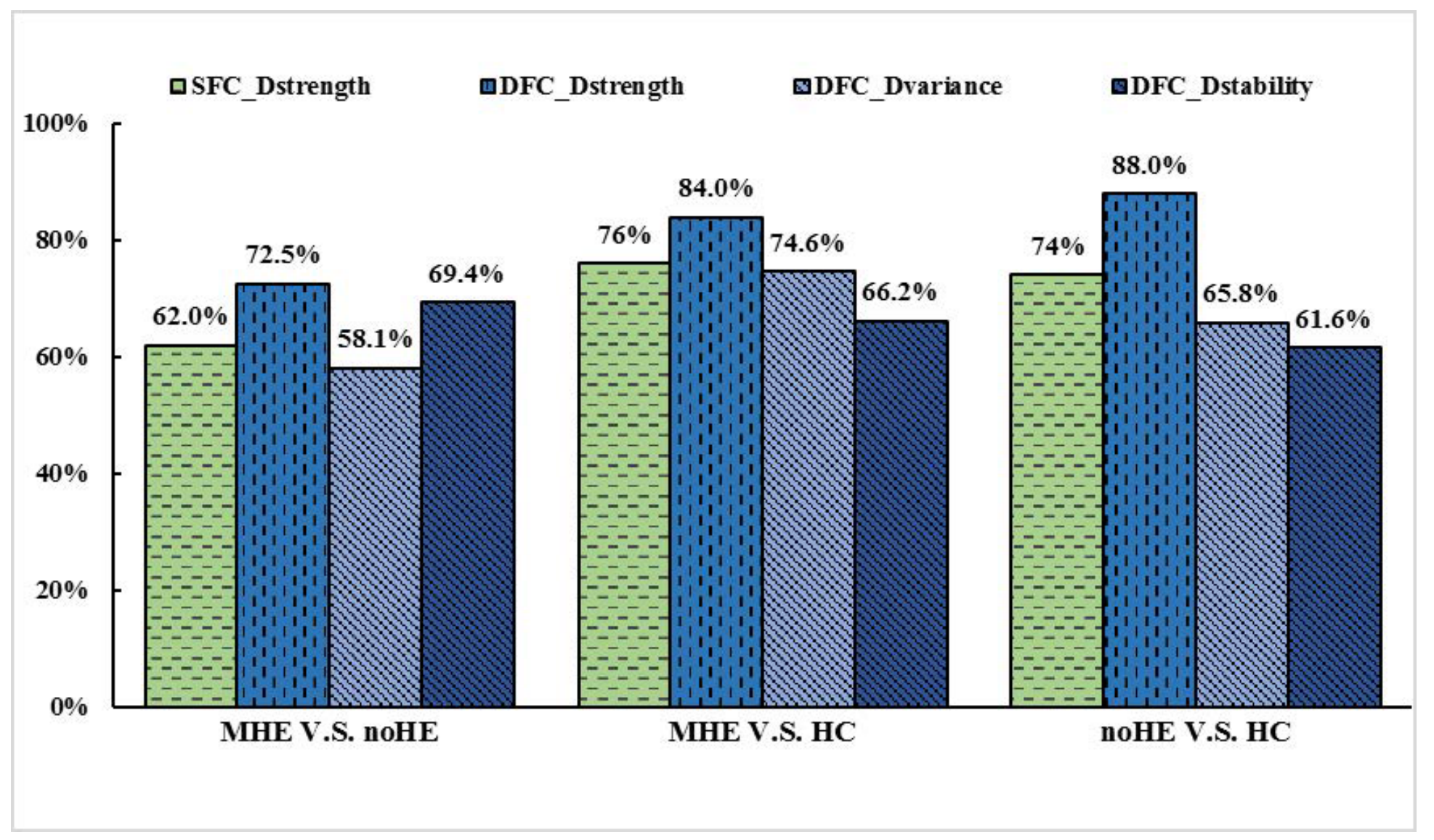

Figure 2

The classification results based on different features (chance level: 50\%) Among the three dynamic features, DFC-Dstrength achieved best accuracy (MHE vs. noHE: $72.5 \%$, MHE vs. HCs: $84 \%$ and noHE vs. HCs: $88 \%)$. Compared to static features, the classification accuracies of DFC-Dstrength feature were improved by $10.5 \%, 8 \%$ and $14 \%$ for MHE vs. noHE, MHE vs. HCs, and noHE vs. HCs respectively. MHE, minimal hepatic encephalopathy; noHE, cirrhotic patients without any hepatic encephalopathy; HCs, healthy controls; DFC, dynamic functional connectivity.

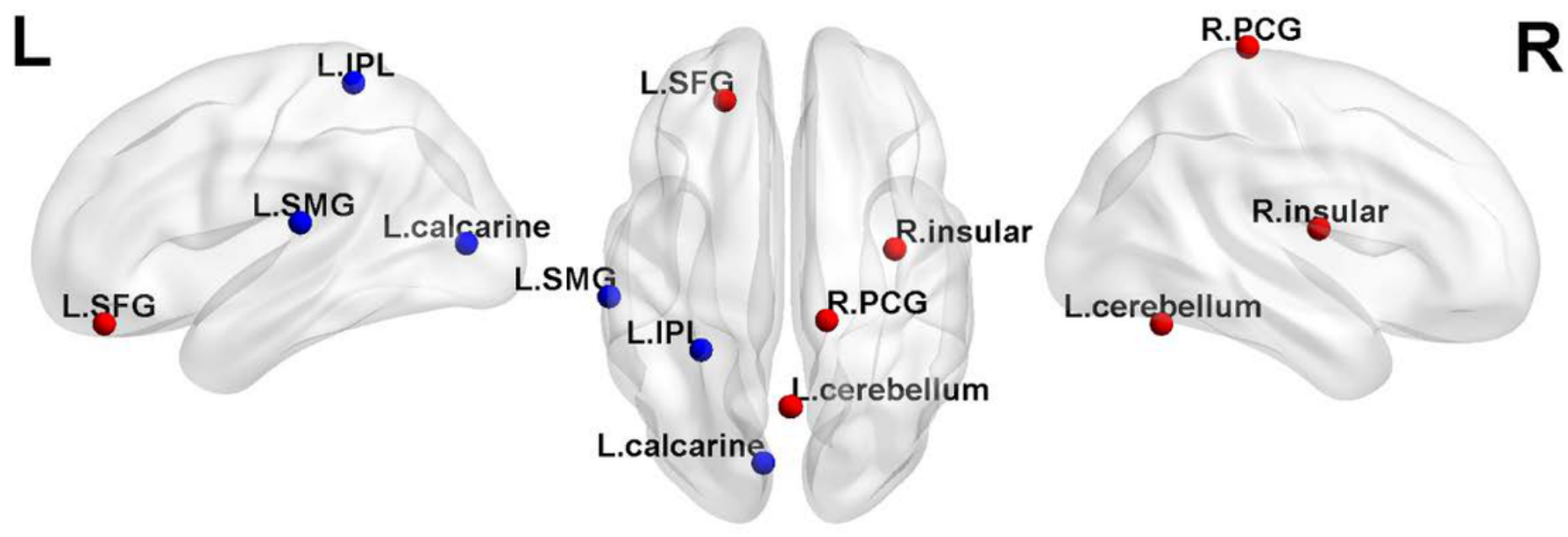

Figure 3 
Spatial locations of discriminant features based on DFC-Dstrength features in MHE patients as compared to noHE group. Seven nodes were identified as the most discriminant features to classify MHE from noHE, including left inferior parietal lobule (IPL), left supramarginal gyrus (SMG), left calcarine, left superior frontal gyrus (SFG), left cerebellum, right postcentral gyrus (PCG) and right insula. The red and blue balls indicate relatively increased and decreased functional connectivity, respectively. MHE, minimal hepatic encephalopathy; noHE, cirrhotic patients without any hepatic encephalopathy; DFC, dynamic functional connectivity. 